\title{
Higgs production @ NNLO: Up-to-date predictions for inclusive cross-sections and differential distributions in different decay channels
}

\author{
Stefan Bucherer* \\ ETH Zurich, ITP \\ E-mail: stefabu@itp.phys.ethz.ch
}

In this talk I will present up-to-date predictions for inclusive and exclusive Higgs production cross-sections at hadron colliders. I put emphasis on the estimation of uncertainties of total crosssections and technical difficulties in obtaining distributions.

RADCOR 2009 - 9th International Symposium on Radiative Corrections (Applications of Quantum Field Theory to Phenomenology)

October 25-30 2009

Ascona, Switzerland

${ }^{*}$ Speaker. 


\section{Introduction}

The detection or exclusion of the Higgs boson is the paramount purpose of ongoing collider experiments at the Tevatron and the LHC. Since the Higgs boson will be produced very rarely - if at all - the expected rate must be predicted very precisely. Furthermore, the precise measurement of the Higgs cross-section might distinguish between different Beyond the Standard Model models.

It is well known that the main production channel at hadron collider - gluon fusion - suffers from large higher order corrections in perturbative QCD. The next-to-leading order (NLO) QCD corrections to the inclusive cross-section were computed already in the nineties [1-3]. While the corrections at NLO are known with the exact mass dependence due to massive quarks in the loops [3-6], next-to-next-to-leading order (NNLO) corrections are only known in the approximation of an infinitely heavy top quark and vanishing Yukawa couplings for all other quarks [7-9]. Recently, sub-leading terms in the top mass have been computed [11-15] and verified the excellent agreement with the large top mass approximation.

The remaining theoretical uncertainty at NNLO is estimated by varying the renormalization and factorization scales to be around $10 \%$. At this precision, electroweak effects become important. The leading light fermion two-loop contribution has been computed in $[16,17]$ and higher order corrections have been studied in [18-20]. Real radiation contributions containing weak gauge bosons have been computed in [21].

Differential cross-sections including decays of the Higgs boson are crucial for experimental searches. In [10,22-25] distributions at NNLO in the heavy mass approximation have been computed.

In order to obtain up-to-date predictions for Higgs production cross-sections we have merged two independent Monte Carlo codes (FEHIP [22] and HPro [26]) into a new code, FeHIPRo [27]. Additionally, we have augmented the computation with electroweak corrections from [20] and [21] and added new features to be described in the following sections.

\section{Finite mass effects at NLO}

We start with a review of the full mass dependence at NLO summarizing [26]. Although diagrams containing a top quark in the loops dominate, the bottom quark contribution cannot be ignored for Higgs masses $<200 \mathrm{GeV}$. For studying the bottom quark effects in more details, we distinguish 'top-only', 'bottom-only' and 'top-bottom' contributions, the interference of top and bottom loops. The investigation of the higher order corrections for these different contributions (see left-hand side of upper row in Figure 1) shows that they are not universal, i.e.

$$
\begin{aligned}
\sigma^{\mathrm{NLO}} & =K_{\mathrm{t}+\mathrm{b}}^{\mathrm{NLO}} \sigma_{\mathrm{t}+\mathrm{b}}^{\mathrm{LO}}=K_{\mathrm{t}}^{\mathrm{NLO}} \sigma_{\mathrm{t}}^{\mathrm{LO}}+K_{\mathrm{t} \times \mathrm{b}}^{\mathrm{NLO}} \sigma_{\mathrm{t} \times \mathrm{b}}^{\mathrm{LO}}+K_{\mathrm{b}}^{\mathrm{NLO}} \sigma_{\mathrm{b}}^{\mathrm{LO}} \\
& \neq \sigma_{\mathrm{fac}}^{\mathrm{NLO}}=K_{\mathrm{t}}^{\mathrm{NLO}}\left(\sigma_{\mathrm{t}}^{\mathrm{LO}}+\sigma_{\mathrm{t} \times \mathrm{b}}^{\mathrm{LO}}+\sigma_{\mathrm{b}}^{\mathrm{LO}}\right) .
\end{aligned}
$$

However, since the bottom contributions are small in size as demonstrated on the right-hand side of the upper row in Figure 1, $\sigma_{\mathrm{fac}}^{\mathrm{NLO}}$ still provides a rather accurate approximation. 

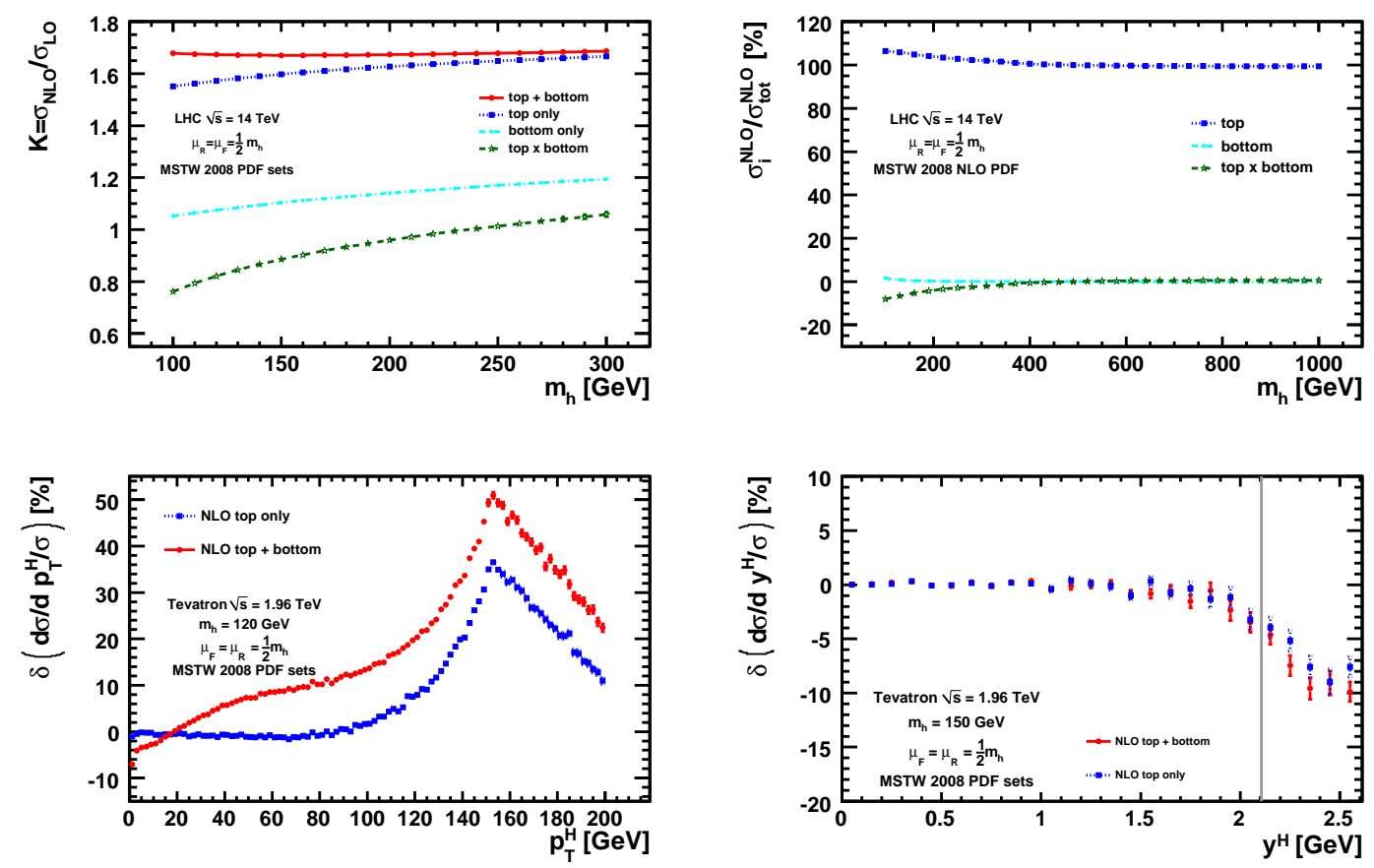

Figure 1: Upper row: On the left: $\mathrm{K}$ factors for top+bottom, top-only, bottom-only and top-bottom interference contributions at NLO for LHC. On the right: Ratio in percentage of top-only, bottom-only and top-bottom interference components with respect to the total cross-section at NLO, for LHC. Lower row: On the left: Relative difference with respect to $m_{\mathrm{t}}=\infty$ approximation for Higgs transverse momentum at the Tevatron, $m_{H}=120 \mathrm{GeV}$. On the right: Relative difference with respect to $m_{\mathrm{t}}=\infty$ approximation for Higgs transverse momentum at the Tevatron, $m_{H}=150 \mathrm{GeV}$.

Next, we study the influence of mass corrections to the shape of the Higgs transverse momentum and the Higgs rapidity. For now and for future reference we introduce the following notation:

$$
\sigma_{(\mathrm{N}) \mathrm{NLO}}^{m_{\mathrm{top}}=\infty}=\sigma_{\mathrm{LO}}^{\text {top-only }} \times \lim _{m_{\mathrm{top}} \rightarrow \infty}\left(\frac{\sigma_{(\mathrm{N}) \mathrm{NLO}}^{\text {top-only }}}{\sigma_{\mathrm{LO}}^{\text {top-only }}}\right), \quad \delta X^{i}=\frac{X^{i}-X^{m_{\mathrm{top}}=\infty}}{X^{m_{\mathrm{top}}=\infty}} .
$$

The transverse momentum distribution (left-hand side of lower row in Figure 1) is affected rather strongly in the large transverse momentum region while the difference is very moderate in the low momentum region. Note that the $p_{\mathrm{T}}$ distribution is effectively leading order. The rapidity distribution (right-hand side of lower row in Figure 1) is only affected in the high rapidity region where only a few events occur. The grey line indicates the rapidity above which less than $10^{-3}$ of the events occur.

\section{Inclusive cross-section at NNLO}

At NNLO, the full mass dependence is not known and existing codes [22,25] compute only the large top mass approximation. We have used our implementation of the massive calculation at NLO 
to improve the prediction of the Monte Carlo code FEHIP. We have also added light fermion two loop contributions containing electroweak gauge bosons and QCD corrections as computed in [20] in terms of an effective theory for $m_{H}=0$. Furthermore, real radiation contributions involving weak gauge bosons [21] are included.

We compare the cross-section including the full mass dependence through NLO with (dashed lines) and without (solid lines) electroweak corrections on the left-hand side of Figure 2. From this plot it is obvious that electroweak contributions are at least as important as the finite mass effects at NLO.
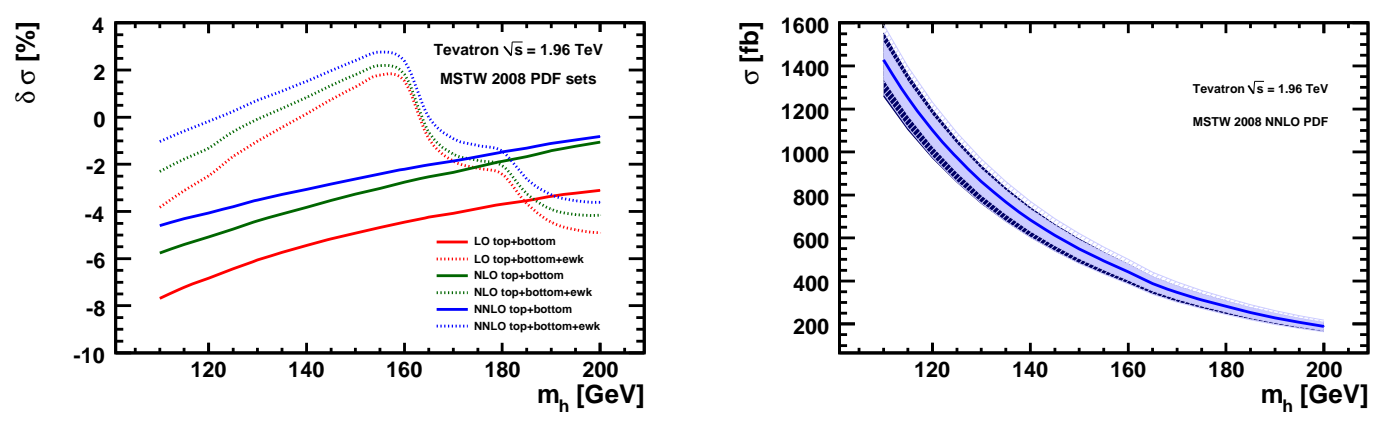

Figure 2: Best predictions for cross-sections at Tevatron. Left: Deviation from $m_{\mathrm{t}}=\infty$ limit of different approximations. Right: Cross-section with band from scale variation (dark blue) and PDF uncertainty (solid light blue band) and PDF $+\alpha_{s}$ uncertainty (shaded light blue band).

Apart from truncated higher order corrections whose size is commonly estimated by varying the renormalization and the factorization scales, the precise knowledge of the cross-section is limited by the uncertainty in the parameterization of the parton densities. In our work we use the MSTW 2008 PDF sets which include error sets for estimating the PDF uncertainty. Rather than assuming a fixed $\alpha_{s}\left(m_{Z}\right)$, the MSTW group uses $\alpha_{s}\left(m_{Z}\right)$ as an additional fitting parameter and one has to consider the combined $\mathrm{PDF}+\alpha_{s}$ error estimate for determining the MSTW uncertainty.

We have organized the computation such that cross-sections for different error sets (for fixed $\alpha_{s}$ ) can be computed simultaneously, thus reducing the computational time considerably.

On the right-hand side of Figure 2 we compare the $\mathrm{PDF}+\alpha_{s}$ uncertainty (shaded light blue band) with the scale uncertainty (dark blue band) for central value $\mu=\mu_{R}=\mu_{F}=m_{H} / 2$ and variation between $\mu=m_{H} / 4$ and $\mu=m_{H}$. While the scale uncertainty is approximately $+9 \%$ and $-12 \%$ over the Higgs mass range $110 \mathrm{GeV}<m_{H}<200 \mathrm{GeV}$ at the Tevatron, the PDF $+\alpha_{s}$ uncertainty at the $90 \%$ C.L. increases from approximately $\pm 11 \%$ for low Higgs masses to $+15 /-$ $13 \%$ at $m_{H}=200 \mathrm{GeV}$.

In summary, we assess the remaining uncertainties on the cross-section as follows: 1 . PDF+ $\alpha_{s}$ uncertainty: $8-15 \%$. 2. Scale variation: $\approx 10 \%$. 3. Resummation effects: As discussed in [8], the scale choice $\mu=m_{H} / 2$ effectively reduces the effect of large logarithms. The comparison to predictions including resummation [28] exhibits a deviation of less than 3\% for Higgs masses smaller than $200 \mathrm{GeV}$. 4. Finite mass effects at NNLO: $<1 \%$ [11-15]. 5. Unknown NNLO coefficient $C_{2 w}$ for mixed QCD-electroweak corrections: $<0.1 \%$ [20]. 


\section{Differential cross-section at NNLO}

The main decay search channels for Higgs production in gluon fusion are $h \rightarrow \gamma \gamma, h \rightarrow$ $W W \rightarrow \ell v \ell v$ and $h \rightarrow Z Z \rightarrow \ell \ell \ell^{\prime} \ell^{\prime}$. The former two channels have been implemented before in FEHIP while the latter channel has only been added in the newest version FEHIPro. In addition, the interference from $h \rightarrow Z Z \rightarrow \ell v \ell v$ where the two charged (same flavor) leptons stem from one $Z$ has been added.

The NNLO computation in FEHIPRO is performed using the method of sector decomposition and a separate adaptation for each sector is required for a satisfactory convergence. However, convergence for the inclusive cross-section does not lead automatically to a good convergence for distributions obtained by histogramming. This can be understood easily: while the Vegas algorithm optimizes the grid for the inclusive cross-section it will mostly sample the regions with the largest contributions. These regions are typically the ones with Born-like kinematics. For distributions, we are more interested in the regions with real kinematics.

To overcome this problem we have introduced a phase-space discriminant,

$$
x_{\mathrm{discr}}=\frac{p_{\mathrm{T}}^{h}}{m_{H}} \cos \theta_{34}
$$

where $\theta_{34}$ is the angle between the two additional final state partons in a double real event $\left(\theta_{34}=0\right.$ if there are less than two final state partons). We then use a modified vegas algorithm from the Cuba library [29] to sample also a distribution in $x_{\text {discr }}$, thus forcing the algorithm to sample the relevant phase space more often and improve convergence for distributions.

In the upper row of Figure 3 we compare the distributions in the difference of photon pseudorapidities, $Y^{*}$, with and without this phase space discriminant. The running time for the computation was approximately the same for both results.

Finally, we assess the effects from finite mass dependence through NLO and the effects from electroweak contributions. In Figure 3 we present a distribution in $Y^{*}$ without selection cuts as well as a distribution in the average photon transverse momentum with selection cuts applied. Except for rare events in the large $p_{\mathrm{T}}^{\text {avg }}$ region, the shape is not affected at all.

\section{Summary}

We have presented new results for Higgs production through gluon fusion at hadron collider obtained with the Monte Carlo code FeHIPro. We have assessed the uncertainties on the inclusive cross-section to be around $10 \%$ from higher orders and of the same order from uncertainties in the PDF parameterization and the choice of $\alpha_{s}$. Other uncertainties are small and well under control.

We have shown that deviations from the large mass approximation due to finite mass corrections are negligible for most distributions in kinematic observables.

In order to improve the convergence for histograms in Monte Carlo computations we have introduced the concept of a phase space discriminant.

Acknowledgements: I am particularly grateful to my collaborators, Charalampos Anastasiou, Radja Boughezal, Zoltan Kunszt, Frank Petriello and Fabian Stöckli. 

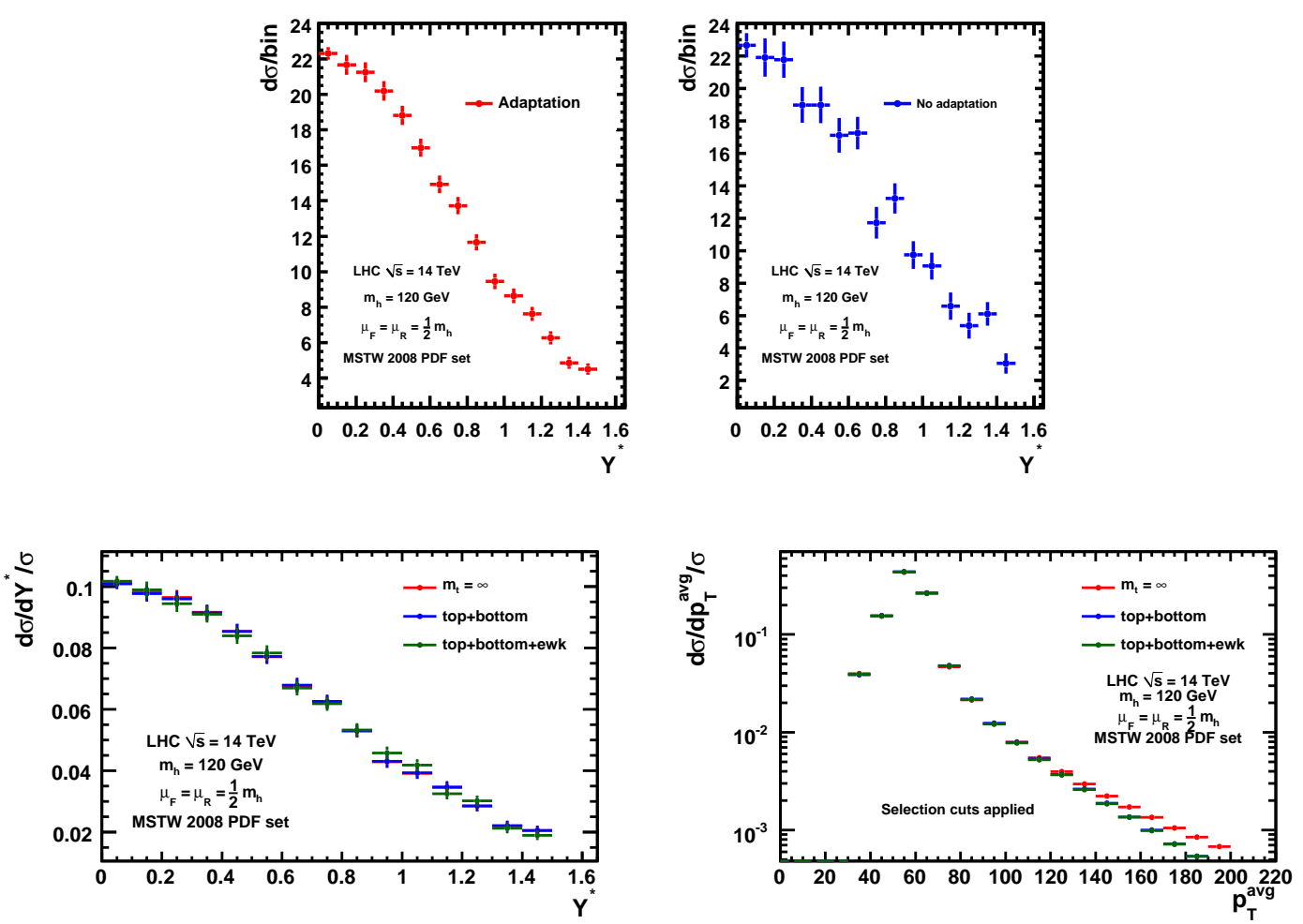

Figure 3: Upper row: Left: $Y^{*}=\left|\eta^{a}-\eta^{b}\right| / 2$ distribution with adaptation to phase space discriminant. Right: $Y^{*}$ distribution without additional adaptation to phase space discriminant. Lower row: Comparison of $m_{\mathrm{t}}=\infty$ approximation with full mass dependence through NLO with and without electroweak contributions. Left: Shape for $Y^{*}$ distribution. Right: Shape for $p_{\mathrm{T}}^{\text {avg }}$ distribution.

\section{References}

[1] A. Djouadi, M. Spira and P. M. Zerwas, Phys. Lett. B 264, 440 (1991).

[2] S. Dawson, Nucl. Phys. B 359, 283 (1991).

[3] M. Spira, A. Djouadi, D. Graudenz and P. M. Zerwas, Nucl. Phys. B 453, 17 (1995) [arXiv:hep-ph/9504378].

[4] R. Harlander and P. Kant, JHEP 0512, 015 (2005) [arXiv:hep-ph/0509189].

[5] C. Anastasiou, S. Beerli, S. Bucherer, A. Daleo and Z. Kunszt, JHEP 0701, 082 (2007) [arXiv:hep-ph/0611236].

[6] U. Aglietti, R. Bonciani, G. Degrassi and A. Vicini, JHEP 0701, 021 (2007) [arXiv:hep-ph/0611266].

[7] R. V. Harlander and W. B. Kilgore, Phys. Rev. Lett. 88, 201801 (2002) [arXiv:hep-ph/0201206].

[8] C. Anastasiou and K. Melnikov, Nucl. Phys. B 646, 220 (2002) [arXiv:hep-ph/0207004].

[9] V. Ravindran, J. Smith and W. L. van Neerven, Nucl. Phys. B 665, 325 (2003) [arXiv:hep-ph/0302135]. 
[10] C. Anastasiou, K. Melnikov and F. Petriello, Phys. Rev. Lett. 93, 262002 (2004) [arXiv:hep-ph/0409088].

[11] R. V. Harlander and K. J. Ozeren, Phys. Lett. B 679 (2009) 467 [arXiv:0907.2997 [hep-ph]].

[12] A. Pak, M. Rogal and M. Steinhauser, Phys. Lett. B 679 (2009) 473 [arXiv:0907.2998 [hep-ph]].

[13] R. V. Harlander and K. J. Ozeren, JHEP 0911 (2009) 088 [arXiv:0909.3420 [Unknown]].

[14] A. Pak, M. Rogal and M. Steinhauser, arXiv:0911.4662 [Unknown].

[15] R. V. Harlander, H. Mantler, S. Marzani and K. J. Ozeren, arXiv:0912.2104 [Unknown].

[16] U. Aglietti, R. Bonciani, G. Degrassi and A. Vicini, Phys. Lett. B 595 (2004) 432 [arXiv:hep-ph/0404071].

[17] U. Aglietti, R. Bonciani, G. Degrassi and A. Vicini, arXiv:hep-ph/0610033.

[18] S. Actis, G. Passarino, C. Sturm and S. Uccirati, Phys. Lett. B 670 (2008) 12 [arXiv:0809.1301 [hep-ph]].

[19] S. Actis, G. Passarino, C. Sturm and S. Uccirati, Nucl. Phys. B 811 (2009) 182 [arXiv:0809.3667 [hep-ph]].

[20] C. Anastasiou, R. Boughezal and F. Petriello, JHEP 0904 (2009) 003 [arXiv:0811.3458 [hep-ph]].

[21] W. Y. N. Keung and F. Petriello, arXiv:0905.2775 [hep-ph].

[22] C. Anastasiou, K. Melnikov and F. Petriello, Nucl. Phys. B 724, 197 (2005) [arXiv:hep-ph/0501130], http://www.phys.hawaii.edu/ kirill/FEHiP.htm.

[23] C. Anastasiou, G. Dissertori and F. Stockli, JHEP 0709, 018 (2007) [arXiv:0707.2373 [hep-ph]].

[24] S. Catani and M. Grazzini, Phys. Rev. Lett. 98 (2007) 222002 [arXiv:hep-ph/0703012].

[25] M. Grazzini, JHEP 0802, 043 (2008) [arXiv:0801.3232 [hep-ph]].

[26] C. Anastasiou, S. Bucherer and Z. Kunszt, JHEP 0910 (2009) 068 [arXiv:0907.2362 [hep-ph]].

[27] http://www.phys.ethz.ch/ pheno/fehipro/

[28] D. de Florian and M. Grazzini, Phys. Lett. B 674 (2009) 291 [arXiv:0901.2427 [hep-ph]].

[29] T. Hahn, Comput. Phys. Commun. 168 (2005) 78 [arXiv:hep-ph/0404043]. 